\title{
LES PARTICULARITÉS DES LANGAGES PARAVERBAUX DANS L'EXPRESSION DRAMATIQUE DE MARCEL PAGNOL: TOPAZE
}

M." Asunción Garcia LaRrañaga - José ORTzZ Domingo

Universidad de Zaragoza

\begin{abstract}
Qu'est-ce que le theatre? Une espece de machine cybernttique. Au repos, cette machine est cachée derriere un rideau. Mais des qu'on la decouvre, elle se met a envoyer à votre adresse un certain nombre de messages. Ces messages ont ceci de particulier, qu'ils sont simultanes et cependant de rythme different.
\end{abstract}

Roland BARTHES

En 1924 et pendant que Marcel Pagnol surveillait l'étude au lycée Condorcet à Paris, il avait écrit, d'après Raymond Castans - l'ami et le biographe de Pagnol-, les premières répliques d'une pièce intitulée la Belle et la bête. Lui, qui montrait son admiration pour les contes de fées et qui les évoquait dans ses ouvres, comme le signale Marie-José Dubois ${ }^{1}$, avait le pressentiment que cette piece aurait un grand succès. Il désirait donc la faire jouer dans un grand

1 MARIE-JosÉ DUBoIs, Marcel Pagnol, écrivain régionaliste ou universaliste? Thże de doctorat presentee et soutenue publiquement a Dijon, Novembre 1991, p. 275. 
thêâtre, par de grands comédiens ${ }^{2}$. Cependant, c'est Jacques Théry, un des amis de Pagnol, qui inspirera involontairement le changement du titre. Pendant une conversation entre les deux amis à propos du vieux pion resté jusqu'à la fin de la pièce à la pension Muche, Théry s'était trompé et il l'avait appelé Topaze. Pagnol l'avait corrigé, immédiatement, en lui indiquant qu'il s'agissait de Tamise. Mais le nom de Topaze lui plaisait. Et comme il n'était pas satisfait de celui qu'il avait donné au personnage principal, M. Martinet, il décida finalement de le rebaptiser. La pièce, en conséquence, recevra un nouveau titre. $L a$ Belle et la bête deviendra Monsieur Topaze. Mais le jour où Pagnol recevra le premier tirage de l'affiche annonçant la pièce, il trouva que la disposition des lettres du titre n'était pas heureuse. Alors, il décida d'appeler l'œuvre, tout simplement, Topaze ${ }^{3}$.

Nous constatons, cependant, que Marcel Pagnol aurait offert au lecteur et au spectateur des indices qui dévoilent ce que la comédie contient en essence s'il avait conservé le titre originaire. De nos jours, l'influence du cinématographe et de la société productrice Walt Disney ont remis a la mode, précisément, l'un des thèmes littéraires les plus aimés de tous ceux qui apprécient l'expression de la sensibilité et les amours impossibles. Si nous remontons dans le temps, nous rencontrons déjà en Grèce et dans l'épopée mythologique composée par Ovide, les Métamorphoses, l'histoire d'un monstre, nommé Polyfeme, amoureux de la charmante et jolie Galaté. En présentant des variantes, ce thème de kla belle et la bête» est repris à différentes époques et il est devenu, en consequence, un sujet universel. Assurément, il offre toujours des connotations qui concernent, à la fois, les contrastes physiques, la dualité entre la réalité et l'apparence et les contradictions morales innées à l'esprit humain ${ }^{4}$.

2 Marcel Pagnol ne se trompait pas. L'ceuvre aura un succes absolu. A partir du 9 octobre 1928, date de la première au théatre des Variétés, le régisseur mettra l'écriteau *complet» pendant des mois. La pižce a été traduite dans toutes les langues et elle a ette jouée dans tous les pays du monde, a l'exception, paraft-il, de la Chine et de la Turquie (Vid. R. BRIAT, «Le Boulevard», in Encyclopédie du théâtre contemporain, dir. par G. QUEANT. Tome II. Paris, Olivier Perrin, 1959, p. 114). Egalement, elle a té réalisée et filmée plusieurs fois. La première réalisation est daté de 1932. d'après la mise en scène de Louis Gasnier; la deuxième est de 1936. Cette fois, le scénario, l'adaptation et la mise en scène ont été créts par Marcel Pagnol, lui-méme. Depuis sa création, nous connaissons huit versions de cette pizce tournce dans différents pays du monde.

3 Voir RAYmond CASTANS, Marcel Pagnol, biographie. Saint-Amand-Montrond (Cher), Editions Jean-Claude Lattes, 1987, pp. 88-115 et 123.

4 Pour la comparaison entre les Métamophoses d'Ovide et le Fantôme de l'Opéra de Leroux, voir l'article de ANTONIO DOMfNGUEZ DOMINGUEZ, *La bella y la bestia: el Fantasma de la Opera de Leroux y la Metamorfosis $n^{\circ} 13$ de Ovidiow, Cruz Ansata, (Bayamon, Puerto Rico), XVI, 1993, pp. 165-177. 
S'il est vrai que le titre de cette pièce peut, dans ce cas précis, nous fournir des renseignements utiles, il n'est pas moins faux que le thêâtre concentre une variété de langages contenant tout un faisceau de messages. Cornme Roland Barthes l'explique dans «Littérature et signification», la thêâtralité, qui est «une épaisseur de signes», nous est donnée par la polyphonie informationnelle. Il distingue sept informations, que l'on reçoit en même temps à un moment du spectacle, venues du décor, du costume, de l'éclairage, de la place des acteurs, de leurs gestes, de leur mimique et de leur parole. Tandis que certaines de ces informations sont conservées tout au long de la pièce —c'est le cas du décor-, d'autres toument-la parole, les gestes ${ }^{5}$.

En même temps on notera qu'on ne peut pas bien comprendre la signification d'une pièce sans tenir compte qu' «une manifestation théâtrale est un ensemble de signes», comme l'écrivait déjà en 1940 Jindrich Honzl $^{6}$. Il est évident que les signes se présentent rarement isolés. «Selon un nombre quasi infini de combinaisons possibles, ils entretiennent entre eux tous les types de rapports: ils se renforcent, se répettent, se précisent, s'annulent, se corrigent, se contredisent», formant «un amalgame de langages»?. C'est donc le spectateur, s'il s'agit d'une piżce représenté, ou le lecteur, s'il s'agit d'un texte, qui doit procéder au déchiffrement de codes. Puisque nous parlons d'un système de communication, le souci de Tadeusz Kowzan porte sur l'intérêt de déceler des unités et des règles de codage du message. Il présente, de son côté, un inventaire des langages employés au théâtre, en ajoutant à celui dressé par Barthes: maquillage, coiffure, accessoire, musique et bruitage ${ }^{8}$. Cette question sur les informations à propos de cet art visant à représenter une suite d'événements devant un public mérite notre attention, et nous nous proposons de l'analyser dans cette étude centrée sur Topaze. Parmi les différents langages proposés par les critiques antérieurement cités, nous nous intéressons à une étude assez particulière que sont les langages paraverbaux qui nous permettrait ainsi de découvrir la véritable signification de la pièce.

Nous croyons qu'il n'y a pas lieu d'insister ici pour savoir qui est l'émetteur de tous ces messages dans Topaze. C'est pourquoi nous avons choisi le personnage, possédant des marques qualificatives et fonctionnelles, comme porteur de tous les signes propres à l'émetteur anthropomorphe9. Pour l'organisation de

5 Roland Barthes, Essais critiques. Paris, Seuil, Coll. «Points», 1981 ( $2^{\circ}$ edition), p. 258.

6 Cité par G. Girard, R. Ouellet, C. Rigault, L'univers du théâtre. Paris, PUF, Coll. \&Littératures Modernes», 1986 (2édition), p. 21.

7 lbid., p. 22.

8 Ibid., p. 30.

9 Les personnages suscitent l'intérêt de MARCEL PAGNOL, comme lui-même le témoigne à 
notre étude, nous avons réuni les informations tout autour de deux axes référentiels: le monde de l'enseignement et l'univers des affaires municipales.

LE MONDE DE L'ENSEIGNEMENT. HONNETETÉ ET ÉCHEC

Nous observons que les aspects topographiques de l'espace où se déroule tout le premier acte de la pièce se situent à l'intérieur de la pension $\mathrm{Mu}$ che. D'un point de vue objectif et général, elle est décrite par Mlle. Suzy, une jeune femme soucieuse de l'éducation de son neveu, comme un espace qui se compose de «cinq ou six caves autour d'un puits» ${ }^{10}$. Mais la partie, la plus importante sans doute de la configuration spatiale est représentée par une salle de classe . Dans une présentation soignée, on nous donne les indications scéniques comportant d'abord la distribution des meubles: devant la chaire, il y a deux rangées de bancs d'écoliers, séparées par une allée. Toute la richesse des matériaux est gardદe dans une armoire vitrée où l'on aperçoit très peu de vieux objets hétéroclites. Il faut souligner l'existence de deux animaux empaillés. L'un, enfermé dans l'armoire, est un perroquet; celui-ci peut être un symbole du mâ̂tre de ce petit microcosme, le professeur, étant donné qu'il est capable d'utiliser la parole pour s'exprimer. La ressemblance renferme généralement une nuance péjorative, car il s'agit d'employer la langue pour répéter systématiquement les mêmes idées comme un automate. L'autre animal est un écureuil. Représentation de la vitalité et de l'agilité, ce petit mammifere, placé sur l'armoire, domine la classe de même qu'il domine la forêt du haut de son arbre. Une des hypothèses concernant son symbolisme dans la pièce serait qu'il pourrait peut-être exprimer la liberté individuelle. Il faut mettre en lumière qu'il occupera toujours une place privilegiée. Il s'ensuit que Topaze, quand il est renvoyé de la pension pour n'avoir pas voulu se soumettre aux mancuvres malhonnêtes proposées par Muche, reprend toutes ses affaires sauf l'écureuil, qu'il dépose délibérément sur l'estrade 11 . On pourrait, donc, considérer l'écureuil comme une certaine revendication de la liberté de Topaze, laquelle est un embleme de pleine vie personnelle et professionnelle.

PIERRE LhOSTE:» La seule chose importante est la creation de personnages (qui) ont plus d'importance que l'intriguex (Citation extraite de la thèse de MARIE-JOSE DUBOIS, op. cit., p. 21).

10 Marcel Pagnol, Topaze. Paris, Fasquelle, Coll. «Le livre de Pochex, 1966, p. 49.

11 lbid., p. 92. 
Cependant l'essentiel dans cet espace, fermé sur lui-même, porte sur les éléments du décor. A côté des cartes de géographie et des tableaux des poids et mesures qui tapissent les murs, et, situés exactement sur les tableaux - l'endroit le plus élevé de nouveau -, divers proverbes se détachent en grosses lettres. Sans doute, le contenu moral de ces dictons possède-t-il une force que le professeur veut transmettre aux élèves, en ratifiant ses propres idées: «Pauvreté n'est pas vice», «Il vaut mieux SOUFFRIR le mal que de le FAIRE», «L'oisiveté est la MËRE de TOUS LES VICES», «Bonne renommée vaut MIEUX que ceinture dorée». Et au centre, justement à la place d'honneur, c'est-à-dire au-dessus de la chaire, on lit un autre proverbe écrit en lettres majuscules: «L'ARGENT NE FAIT PAS LE BONHEUR ${ }^{12}$. Voilà l'étendard que portera d'abord Topaze, le professeur de cette classe, comme symbole de sa propre cause.

Au début du siècle, André Antoine, ami de Marcel Pagnol, avait proposé qu'on situe d'abord le décor sans aucune preoccupation du spectateur ni des étenements, car «c'est le milieu», a son avis, «qui determinait les mouvements des personnages, et non les mouvements des personnages qui déterminaient le milieu» ${ }^{13}$. Dans cette optique, dans Topaze le décor apparaît, justement, comme une prédétermination du personnage principal. Enfermé dans un monde clos, celui-ci conservera ses idéaux et maintiendra ses rêves jusqu'au moment même de son renvoi. Néanmoins, le décor offre, de même, à l'aide de la langue écrite emploýe dans ces vérités de sagesse populaire que sont les proverbes, une projection de Topaze, tout en communiquant au récepteur les traits essentiels de sa pensée.

En se questionnant sur l'importance du costume au theâtre, Roland Barthes affirme qu'il possède kune nature fonctionnelle». Le critique essaie de définir son genre de prestations, et il reconnaît d'abord que le costume doit être un argument. Ayant une forte valeur sémantique et comportant comme élément de base le signe, il peut communiquer des idées, des connaissances ou des sentiments ${ }^{14}$.

C'est une ressemblance bien analogue qui unit Topaze à Tamise ${ }^{15} \mathrm{du}$ point de vue du costume. Dés sa première apparition sur scène, Topaze porte

12 Ibid., p. 11.

13 L'univers du théâtre, p. 84.

14 Op. cit., p. 58.

15 RaYmond CASTans raconte que Pagnol a connu au college Tarascon, en 1915, un vieux pion retraité, M. Boucoiron, rappelé en service, kune espezce de M. Soupe de l'enseignement», dont il s'est inspire pour composer son personnage de Tamise (op. cit., p. 50). 
«un col droit, très haut, en celluloïd, une cravate misérable, une redingote usée et des souliers à boutons» ${ }^{16}$. De Tamise, nous savons qu'il a «visiblement le même tailleur que Topaze» ${ }^{17}$. D'abord, les vêtements et les autres effets de l'habillement reflètent la réalité professionnelle et économique de ces deux personnages. Appartenant au monde de l'enseignement, ils sont obligés de porter une espèce d'uniforme qui les caractérise tous. Il exprime la dignité du métier et une certaine solennité, la couleur noire aidant à souligner le caractère sérieux de la profession. Cependant, les bas salaires les obligeaient de garder pendant des années les mêmes vêtements, ce qui contribuait à présenter une apparence pauvre. Pagnol recourt, donc, ici à un type du personnage théâtral, lequel est marqué par un costume spécifique servant à son identification. La valeur symbolique provient d'un code extrafictionnel socioculturel. L'analogie entre les costumes de Topaze et de Tamise nous permet d'établir ainsi des rapports très étroits entre ces deux personnages, qui seront d'ailleurs d'excellents amis. Remarquons que cette identification ou dualité, qui apparaît dans la première partie de la piżce, subira une mutation à mesure que Topaze changera peu à peu de personnalité.

Si cette ressemblance est significative, la disparite des costumes de ces deux personnages par rapport a ceux de Muche et Emestine nous fournit des renseignements sur les differents rôles et fonctions que tous ces personnages auront dans la pièce dramatique. M. Muche, le directeur de la pension, porte un «col cassé et un costume neuf marron clair» ${ }^{18}$. La symbolique de la couleur exprime déjà sa supériorité par rapport à l'infériorité de la couleur noire. Et, en plus, sa situation économique aiste se voit dans la différence de forme du costume et dans la nouveauté de celui-ci. Sa fille Ernestine, qui est le calque de son père, est «vêtue avec une élégance bon marchê» ${ }^{19}$. C'est-à-dire qu'elle a l'allure de la bourgeoise qu'elle est, mais elle n'a pas encore l'argent suffisant pour pouvoir le faire voir aux autres. En offrant un aspect agréable et en s'habillant à la mode, Ernestine ne peut pas encore acheter ses habits dans les boutiques de renom. Ainsi son rêve est de pouvoir un jour se promener avec des manteaux de fourrure. Nous constatons que la réalité socio-économique de Muche-Ernestine est tout autre que celle de Topaze-Tamise. Elle exprime la situation et les idées d'une classe sociale déterminée, la moyenne bourgeoisie, préoccupée spécialement par le monde de l'apparence. Par l'opposition entre les costumes, nous constatons, alors, que Muche et Ernestine

\footnotetext{
16 Marcel Pagnol, p. 13.

17 Ibid., p. 29.

18 Ibid., p. 20.

19 Ibid., p. 13.
} 
interviendront dans la pièce en remplissant la fonction d'opposants au désir de Topaze. Tandis que Tamise est le seul qui réalisera, par contre, la fonction d'allie ou d'adjuvant.

$$
\text { *** }
$$

Il est intéressant de relever que parmi ces langages paraverbaux, l'accessoire se situe, pour les auteurs du livre L'univers du théatre, entre le décor et le costume ${ }^{20}$. L'analyse de certains objets appartenant aux différents personnages confirme, en principe, la différence que nous venons d'établir. En faisant un petit inventaire des accessoires, nous notons que Muche est le seul qui porte «une grosse bague au doigt et une chaîne de montre éclatante» ${ }^{21}$. Ces objets personnels et «décoratifs» nous renseignent sur son désir d'exhiber sa richesse, un pouvoir économique qui manque de distinction. Cette vulgarité a probablement une connotation temporelle. Elle reflete le moment present, le besoin de cette société française de l'entre-deux-guerres de vivre dans l'opulence, et de juger d'après ce qui paraît extérieurement. Marcel Pagnol n'a pas été le seul à dénoncer cette réalité, il est significatif que Bourdet, Anouilh, Aymé et Marceau expriment le même souci en critiquant une société qui vit des apparences et de l'apparat. D'après Michel Corvin, cette reaction est trop generale et elle est représentée par des écrivains très différents pour être attribuce à un esprit de revanche ${ }^{22}$. Contrairement à Muche, Tamise et Topaze ont des lorgnons à cordon et ce dernier garde dans sa poche une montre énorme et presque sphérique, un oignon. Le caractère ancien de la montre et les lorgnons démodés prouvent leur appartenance au passé. Ce qui est hors de doute, c'est que le chapeau melon de Topaze augmente le ridicule de cette figure avec une longue barbe noire qui se termine en pointe. Quand on se réfère à lui, on le désigne dédaigneusement comme: «Ce malheureux barbu en chapeau melon ${ }^{23}$. Cependant, le comble d'un ridicule repoussant nous est offert par ce chapeau de paille aux bords gondolés de Panicault. Ce professeur, qui a largement dépassé la soixantaine, montre des dents aussi vertes que la couleur de son parapluie et roule entre ses doigts une cigarette fripee. Dans ce cas, le ridicule va de pair

20 Op. cit., p. 68.

21 Marcel pagnol, p. 20.

22 MiCHEL CORVIN, «Le Boulevard en Question», Le theâtre en France, sous la direction de Jacqueline de Jomaron. Tome 2. De la Révolution d̀ nos jours. Paris, Armand Colin, 1989, pp. 363364.

23 MARCel PAgnOL, p. 122. 
avec l'absurdité de sa pédagogie. L'auteur critique la «méthode Panicault» qui consiste, en cas de doute, à punir l'élève dont la tête revient la première à l'esprit du maître. S'il y a un objet que Topaze désire obtenir plus que toute autre chose ce sont les palmes académiques. Ce qui peut paraître d'abord une ambition démesurée, propre à un arriviste, comme l'indique Tamise ${ }^{24}$, n'est en réalité qu'un certain orgueil professionnel, la reconnaissance du travail bien fait. Remarquons que tous ces objets cessent d'être neutres par l'importance qu'on leur accorde dans cette pièce dramatique.

Il y a aussi évidemment toute une série de petits accessoires professionnels, nécessaires au travail de l'enseignant. Si Tamise et Ernestine portent des serviettes sous le bras, Topaze, soigné et méticuleux, possède toute une variété d'objets: flacons, boîtes de craie, carnets de notes, porte-plumes, essuie-plumes, etc. La fonction réaliste que ces accessoires accomplissent contribue à la vraisemblance du spectacle 25 . Mais la portée générale de ces objets est dépassé par les livres que Topaze utilise, d'habitude, dans ses cours. C'est en particulier aux livres de fantaisie que le personnage s'intéresse, jusqu'au point de vouloir faire un récolement général à ses moments perdus ${ }^{26}$. Le caractère de héros appartenant au passé s'intègre parfaitement à cet univers fantaisiste et imaginatif, qui permettra à Topaze de vivre dans un monde irreel:

J'Etais le vaillant chevalier, choisi pour combattre le monstre et delivrer la beauté prisonnière... Je vivais dans un rêve, dans une atmosphère de poésie et d'extravagance...27

En outre, afin de nous faire connaître, à l'avance, la signification profonde de la comédie, nous avons observé une troisième référence au domaine de la zoologie. Il s'agit cette fois de l'allusion à un putois que Topaze montre à ses élèves pour «illustrer une leçon sur les ravageurs de la basse-cour ${ }^{28}$. A la manière d'une prémonition, annonçant déjà au premier acte ce qui va arriver au cours de l'histoire dramatique, le putois devient véritablement l'articulation de la pièce. C'est aussi à un putois que Castel, le prévaricateur, veut écraser la

24 lbid., p. 31.

25 Petr Bogatyrev, distingue dans son etude «Les signes du theltrew, Poetique n. 8,1971 , pp. 517-530, entre des signes de signe d'objet et des signes d'objet. En admettant que les premiers sont beaucoup plus frequents sur la scène que les signes d'objet, il souligne que tous les signes n'ont pas une fonction de representation; «par exemple, un acteur jouant un homme affame peut montrer qu'il mangue du pain en soi, et non pas du pain comme signe, disons, de la pauvreté (p. 518).

26 MARCEl Pagnol, p. 23.

27 lbid., p. 175.

28 lbid., p. 23. 
tête sous son pied ${ }^{29}$. Cependant, l'inoffensif Topaze changera d'idées et de comportement au fur et à mesure qu'il souffrira davantage, pour se présenter finalement comme le putois qui détruira tous ses adversaires.

Finalement, nous voulons tout simplement faire référence à cette attitude corporelle qu' on qualifie de geste, laquelle constitue un nouveau moyen d'expression parmi les langages du théâtre. Accompagnant généralement la parole et la prolongeant parfois pour compléter l'énoncé, le geste s'associe à la parole dans un rapport d'intensité. Il a une fonction didactique parce qu'il ponctue la pensé du personnage, de même qu'il a une fonction lyrique parce qu'il traduit les sentiments de celui-ci ${ }^{30}$. Par exemple, en donnant plus de relief à ce que Topaze éprouve lorsqu'il craint de perdre definitivement Ernestine, on expose toute une gradation ou phases de ses gestes. Is expriment qu'il est tout d'abord «ému», pour passer immédiatement au «très ému» et finalement au «pathétique» ${ }^{31}$. Néanmoins, quand l'espoir habite encore son cour, il se montre d'abord «lyrique» et plus tard «enflamme» ${ }^{32}$. En ces moments de joie, il lisse sa barbe comme signe de satisfaction et reste songeur, en recreant ses propres fantaisies. Plus Topaze est ému, plus Ernestine sourit. Les gestes qu'elle emploie expriment, par contre, le dédain, l'arrogance et la dureté. L'extrême de ce mépris est la gifle qu'elle lui donne, en le repoussant de toutes ses forces ${ }^{33}$. Une nouvelle expression d'orgueil et de mépris se lit chez la baronne, qui déchire le bulletin de son fils et jette les morceaux au nez de Topaze, quand elle n'obtient pas ce qu'elle veut. En se servant de ses gestes, Muche joue aussi avec Topaze, de même qu'Emestine, comme le chat avec la souris. Si, d'habitude, il demeure indifferent et autoritaire, lorsqu'il veut «dompter» Topaze, il le fait souffrir. Entre autres, il utilise un stratageme: il introduit l'angoisse dans l'état d'esprit du professeur. Puisqu'il sait que Topaze attend avec impatience la réponse à sa demande sur les palmes académiques, il fouille, à la fin de la scène, dans la chemise qu'il porte à la main depuis le début. Mais, pour augmenter la tension dramatique et pour ralentir l'action, il fouille et refouille un

29 Ibid., p. 108.

30 L'univers du thêatre, p. 46.

31 MarCel PAGNOL, p. 16.

32 lbid., pp. 17-18

33 Ibid., p. 58. 
long moment, en cherchant toujours. Pour finalement lui dire qu'il a eu les palmes académiques «moralement». Le seul geste de Tamise, qui «hausse les épaules avec indulgence» ${ }^{34}$, est celui qui implique l'acceptation de son ami tel qui est, même s'il doute de pouvoir le faire un jour changer d'avis.

En tant que grand maître de comédies et de vaudevilles, Marcel Pagnol excelle évidemment à dominer tous les procédés du comique. C'est ainsi que chez lui le rire a une fonction sociale. Nous observons que, par un mouvement parallèle à celui qui s'opère dans les mots, les gestes nous font penser, parfois, à une simple mécanique. Le sourire du destinataire provient de l'impression du comique produit par la vision d'un pantin articulé. Selon la formule d'Henri Bergson, c'est «du mécanique plaqué sur du vivant» 35 . C'est ainsi qu'on voit la réaction de Topaze au moment où il écrit au tableau. Un élève lance un morceau de papier roulé qui tombe à côté du professeur. Alors, il «se retourne brusquement, comme mâ par un ressort. Les yeux fermés, la barbe hérissée, il tend son index menaçant vers la gauche de la classe et crie» ${ }^{36}$. Cet effet comique est saisissant parce qu'à l'intérieur de cette figurine comique nous découvrons les manières et les mouvements d'un être vivant.

L'univers des affaires muniCipales. Prévarication et SUCCÈS

Il est vrai que les deux espaces où l'on va voir désormais évoluer les personnages bien que differents sont à la fois complémentaires.

Le premier d'entre eux est le «Boudoir» de Mlle Suzy Courtois. Or cet espace que le spectateur ne pourra découvrir physiquement que dans le deuxième acte y a fait son entrée dans la conscience des gens bien avant. C'est Topaze qui se charge de nous le dévoiler, de nous le peindre oralement dans le premier acte dans les termes suivants: «Elle m'a reçu dans un boudoir des Mille et une nuits. Des étoffes de soie, des tableaux anciens, des coussins par terre. Le tapis était épais, souple, et avec ça - ça a l'air d'une blague - il dépasse sous la porte jusqu' au bas des escaliers» ${ }^{37}$. Or, cet endroit illusoire, digne des plus fins éloges littéraires, qui traduit à première vue l'aisance, la richesse, la

34 Ibid., p. 39.

35 HenRI Bergson, Le rire. Paris, PUF, Coll. «Quadrige», 1983, p. 29.

36 Marcel Pagnol, p. 61.

37 lbid., p. 45. 
finesse et le bon goût, qui a impressionné et contaminé notre personnage, cache de bien rudes réalités. C'est l'endroit où ont lieu les ébats amoureux entre amants; c'est l'endroit où l'on traite et où l'on signe des affaires importantes, mais surtout malhonnêtes; c'est l'endroit où l'on amadoue et où l'on jette le dévolu sur un «prétendu idiot» pour le faire entrer dans des combines extrêmement périlleuses. Un endroit qui émerveille mais qui n'est point merveilleux, car sous l'apparat d'une illusion exotique se cache le mensonge le plus cru et la plus ignoble immoralité.

Le «bureau moderne et tout neuf» qui fait son apparition dans le trosième acte, est pour ainsi dire le prolongement du Boudoir. C'est même Suzy qui nous le fait savoir quand elle annonce à Roger: «Régis vient d'acheter l'immeuble voisin, et tout le premier étage est transformé en bureaux. J'ai fait percer le mur ici» ${ }^{38}$. Ce nouvel espace est en réalité le centre opérationnel, capable de transmettre à lui seul toute la philosophie de ce nouveau monde où Topaze s'est vu plongé. Sous l'élégance de la décoration, du bon gout et de la richesse représentée par les «deux énormes fauteuils de cuir» et «un formidable bureau américain» ${ }^{39}$, le mot et l'idée «Argent» règnent en maître. Aux inscriptions catégoriques comme «Soyez brefs», «le temps, c'est l'argent», «Parlez des chiffres», dejà significatives en elles-mêmes, viennent s'ajouter des éléments du décor, tels que le coffre-fort, symbole de jouissance économique, et l'inscription sur une porte du mot «comptabilite», qui viennent renforcer encore plus cette idée. En réalité, cet endroit, transformé pendant un temps en refuge ou prison de Topaze vis-à-vis de sa propre conscience, peut être considéré comme la plateforme tournante où sont exposés à vif certains maux dont souffre notre société. C'est là où l'on paie les conseils, les commissions, les pots-de-vin, où l'on étouffe la parole et où l'on achète le silence, où l'on contrôle les associés, ou l'on fait du chantage et où l'on prêche le pouvoir de l'argent.

Si l'espace où se meut Topaze dans la pension Muche est étouffant, fermé sur lui-même, où le moindre geste est contrôle, le boudoir et le bureau sont au contraire des espaces ouverts: on voit défiler des gens. Si en réalité, ces deux endroits n'ont pas la même fonction éducative que l'espace de la pension $\mathrm{Mu}$ che, ils se haussent cependant comme la véritable école de la vie. Les enseignements les plus divers y sont professés, et d'une façon si convaincante qu'ils sont capables de transformer le plus ignorant et le plus candide des êtres humains en un véritable et abominable escroc. A ce niveau, nous trouvons à la fin

38 Ibid., pp. 102-103.

39 Ibid., p. 163. 
de la pièce un passage totalement significatif. C'est le moment où Castel-Bénac compare la métamorphose de Topaze avec celle d'un singe:

C'est exactement l'histoire du chimpanzé de ma mère. Quand elle l'a acheté il était maigre, il puait la misère, mais je n'ai jamais vu un singe aussi affectueux. On lui a donné des noix de coco, on l'a gave de bananes, il est devenu fort comme un Turc, il a cassé la gueule à la bonne. Il a fallu appeler les pompiers... ${ }^{40}$.

Il est vrai que cette comparaison n'est point fortuite, que la ressemblance de l'homme et du grand singe est connue de tous. C'est la caricature de l'être méprisable, car la caractéristique essentielle de cet animal est sa force instinctive, incontrôlée et par conséquent dégradante. Cependant, l'importance de cette comparaison ne porte pas sur l'aspect négatif que Castel-Bénac veut mettre en évidence mais, au contraire, sur ce que cache l'image même du chimpanzé. Or, la tradition veut qu'aux visions purement négatives de cet animal viennent s'en superposer d'autres qui exaltent les vertus les plus absolues. L'image que nous avons de Topaze coïncide totalement avec celle qu'ont du singe les indiens: celle «d'un malin magicien, masquant ses pouvoirs, dont le premier est l'intelligence, sous des aspects caricaturaux», ou alors, il est dans la lignée de la tradition chinoise qui le considère comme «un sage initié qui cache sa véritable nature sous une apparence bouffonnew 41 . Intelligence, énergie, savoir cacher ses pouvoirs et les utiliser au moment propice, voila les caractéristiques essentielles du singe et par conséquent de Topaze. En rélité, en voulant traiter de misérable le pauvre Topaze, lui rappelant sa provenance, et en le traitant de chimpanze, Castel-Bénac, inconsciemment loue l'intelligence et le savoir-faire de cet être qui lui a tout, absolument tout volé.

Il est vrai également que les vêtements de même que l'aspect physique des personnages jouent un rôle et non des moindres dans ce monde des affaires.

Dès le premier instant, Suzy apparaît devant nos yeux comme la personnification même de l'elégance. Son upetit chapeau de feutre sur des cheveux

\footnotetext{
40 Ibid., p. 224.

41 Jean Chevalier, Alain Gueerbrant, Dictionnaire des symboles. Paris, Robert Laffont,
} 1982 , p. 886. 
blonds» et son «vison splendide sur une robe très moderne» ${ }^{42}$ causent une grande impression dans l'entourage de la pension Muche et viennent rompre la vulgarité et la monotonie qui caractérisent cet endroit. De même, «les bas tissés de la plus fine soie», les «petits souliers precieux, [...] en peau de gant, ou en peau de serpent» que porte si délicatement Suzy, ou «le parfum qu'elle répand» ${ }^{43}$ sont comme des filtres qui viennent ennivrer Topaze, à tel point qu'il ne demeure plus maître ni de ses idées, ni de ses propres paroles.

Si l'image de Suzy repose tout au long de la pièce dans la lignée de la perfection, celle du «pion galeux» va subir une profonde métamorphose. On le voit se défaire peu à peu de ses vieux haillons, capables comme on le signale, d'être le déguisement parfait des maîtres-chanteurs, où l'appât parfait pour toucher et attendrir les cours sensibles, pour endosser ceux que sa nouvelle classe sociale lui exige. «Le chapeau de fente d'hommes d'affaires» 44 remplace celui de professeur, «les grosses lunettes à monture d'écaille» ${ }^{45}$ font oublier les lorgnons, et «le costume de bon faiseun ${ }^{46}$ enterre à jamais celui kde la distribution des prix ${ }^{47}$. Ce monde, qui attribue à chacun une nouvelle personnalite, modèle également à la perfection la personne. Conscient qu'il doit se débattre dans le monde de l'apparence, à la fois cruel et hostile, et convaincu que pour obtenir la gloire il faut cultiver l'image à tous les niveaux, comme nous le montre l'honorable vieillard, il se lance dans une course effréné pour se defaire à jamais de ces indices ou de ces traces capables de lui rappeler qui il fut et d'où il vint. Ce n'est point étonnant donc, qu'après un séjour relativement court dans ce monde qui l'a adopté, son propre ami Tamise ait du mal a le reconnaître et qu'il le compare à un kacteur de la Comédie Française» ${ }^{48}$, tant il a changé.

En réalité, si les vêtements mettent en evidence une transformation du personnage en question, cette métamorphose ne doit point être vue sous un aspect purement positif. Au contraire, plus la tenue qu'il porte touche la perfection, plus elle semble marquer la déchéance morale du personnage, au point que l'on pourrait affirmer que l'immoralité des gens est directement proportionnelle à leur raffinement dans le costume.

42 Marcel pagnol, p. 47.

43 lbid., p. 46.

44 lbid., p. 139.

45 lbid., p. 165.

46 Ibid., p. 224.

47 lbid., p. 104.

48 Ibid., p. 242. 
Au niveau des accessoires, il faut signaler qu'ils peuvent être classés en trois groupes différents. On a pu constater que certains ont un rapport direct et absolu avec le modernisme et le monde du travail, que d'autres sont le symbole pur et net de l'opulence, tandis que les derniers nous renvoient à l'oisiveté la plus absolue. Mais ces groupes ne sont pas fermés sur eux-mêmes, et cela tout simplement parce que des termes comme TRAVAIL-ARGENT-OISIVETÉ sont dépendants et complémentaires les uns des autres, d'où la possibilité que certains accessoires puissent être à cheval ou s'intégrer dans deux groupes différents. Nous voudrions préciser également qu'à l'intérieur même du groupe certains objets jouissent de connotations particulières.

Bien que les annuaires, le bottin, le carnet, le registre, les lettres, le télephone, les dossiers et le journal soient des accessoires ou des objets propres au monde du travail auquel ils appartiennent, les quatre derniers s'éloignent de leur fonction première. C'est ainsi que les dossiers de l'Agence Topaze semblent être non pas des suivis détaillés des affaires, mais des fiches policières complètes sur les activités douteuses des associés ou des personnes haut placées. Quant aux autres accessoires, ils s'éloignent quelque peu de leur rôle purement communicatif et endossent le masque de l'accusation particulière. Les lettres anonymes, les articles de la Conscience publique, ou les coups de téléphone, sont des moyens par lesquels est déployé le plus vil chantage, coutume habituelle, paraît-il, comme le dit Topaze, dans les régimes démocratiques et autres. Les lunettes, l'étui d'argent, la montre, la perle, les chèques, les liasses de billets, les titres, et la voiture nous renvoient au monde de l'opulence et ont comme fonction de mettre en vedette «l'Argent», cette force qui gouverne le monde, et qui est capable à elle seule d'acheter le confort, la beauté, les amours et la puissance. Nous savons combien Pagnol aimait l'argent et comment il en fut fasciné dès sa plus tendre jeunesse. Ce n'est point étonnant de voir Topaze prêcher son pouvoir afin d'ouvrir les yeux de son bon ami Tamise et lui montrer la terrible réalité des choses:

L'argent peut tout --dit-il, il permet tout il donne tout (...) Regarde ces billets de banque, ils peuvent tenir dans ma poche, mais ils prendront la forme et la couleur de mon désir (...) C'est la force qui gouverne le monde, et ces petits rectangles de papier bruissant, voilà la forme moderne de la force. ${ }^{49}$

Cependant, pour mettre en relief l'obscure provenance de l'argent, Pagnol recourt de façon magistrale à la symbolique des objets, procédé qui lui permet

49 Ibid., p. 247. 
d'établir des correspondances très fines, osées parfois, mais extrêmement riches. C'est ainsi que dans Topaze, «les trafics d'un conseiller municipal malhonnête portent sur des objets répugnants: vespasienne, balayeuses, plaques d'égout, crachoirs, éclairage de l'abattoir, frigorifique de la morgue..., car ils servent sur le plan poétique à évoquer la noirceur de ses activités. „50

Dans le groupe de l'oisiveté, nous intégrerons simplement deux accessoires: le journal illustre et la carte. Le premier de ces objets traduit les nouvelles inquiétudes de Topaze et met en évidence comment le snobisme et la pédanterie sont la nourriture habituelle de cette classe qu'il a faite sienne. Quant à la carte, symbole de liberté et d'évasion, elle prend ici une dimension toute nouvelle. Elle a, certes, une fonction civilisatrice et met en relief la puissance coloniale française; elle manifeste en outre que les affaires vont prendre un nouvel envol, que l'hexagone ne sera plus le centre d'action et que les délibérations importantes s'éloignent du Conseil Municipal et gagnent les bureaux ministériels.

\section{***}

Jean Copeau a signalé comment la musique et le bruitage demeurent comme «des éléments constituants de la représentation thêâtrale» 51 . Les exemples n'abondent pas dans la pièce, mais les deux qui apparaissent sont significatifs. Dans le premier acte nous avons «une boîte à musique qui émet trois notes: ding! ding! dong! $\$ 2$, et dans le troisieme acte trois balayeuses disent: «Tripoteur», «»Tripoteur», «Tripoteur», et chuchotent: «Topaze escroc!», «Topaze escroc!» ${ }^{53}$. La musique et les bruits abandonnent leur fonction première, s'émancipent et deviennent comme des interlocuteurs en lutte avec le personnage. Ces architectures mobiles et évanescentes agissent directement et profondément sur sa sensibilité. La boîte à musique et ses notes mettent en évidence le manque de respect des élèves vis-à-vis de leur maître, tandis que les balayeuses et leurs bruits en plus de contribuer à évoquer une atmosphère, nous montrent cette lutte interne entre le personnage et sa propre conscience, et nous dévoile son état psychologique et son état d'âme.

***

50 MarIE-José DuBoIs, op. cit., p. 214.

51 L'univers du théâtre, p. 76.

52 Marcel pagnol, p. 32.

53 Ibid., p. 176. L'episode de la boíte à musíque et celui des balayeuses sont tirés de faits réels et puisés dans les souvenirs de l'auteur. 
Il est vrai également que les gestes et les mouvements, étant au niveau théâtral un complément important de la parole, émettent en outre de précieux renseignements sur les personnages. A travers les gestes de Régis, $* I l$ montre du doigt», «il frappe sa poitrine», «tend la main brusquement», «Examine les papiers et Topaze minutieusement», «reprend vite les papiers sur la table», «passe devant les invités», «ne fait que regarder la montre», «cligne continuellement l'œil a Suzy» etc.... nous avons une image complete du personnage. Ils mettent en évidence sa brusquerie, sa méfiance et sa mauvaise éducation entre autres.

Le caractère de Suzy est dévoilé également à travers ses gestes. On la voit «servir des cocktails», «se lever et aller vers les invités», koffrir des cigarettes», «serrer les mains de Topaze», «lui montrer ses mains et ses dents», «lui attacher un ruban violet à sa boutonnière», etc., autant de gestes qui montrent son savoirfaire, sa grande diplomatie, mais aussi une dose importante de bienveillance et de sentimentalité. Mais Topaze est sarement le personnage qui se définit le mieux par ses gestes. Eux seuls sont capables de nous montrer les diverses étapes de sa mutation. Dans un premier temps, il est comme perdu dans ce monde qui n'est pas le sien, mal à l'aise, hésitant. On le voit «rougir», «bafouiller», «tousser», «toussoter», «baisser les yeux», «s'asseoir au bord de la chaise», «s'incliner profondément». Une fois introduit dans ce monde, on le voit en lutte avec sa propre conscience, inquiet et craintif: «Il tressaille» quand on frappe à la porte, «il entrebaille la porte de son bureau et ne laisse entrer personne», «il se pince les narines pour répondre au téléphone», «il écarte le rideau avec précaution pour regarder à travers la fenêtre», etc. La troisième phase nous montre déjà un Topaze métamorphose, un Topaze confiant, sâr de lui, et qui a fait siennes les bonnes manières qui régissent son nouveau monde. On le voit baiser la main de Suzy, lui tendre un journal illustre pour qu'elle lise, «offrir une cigarette», «faire attendre Roger et Suzy», «sortir du coffre-fort une liasse de billets», etc., toute une série de gestes qui font preuve de pouvoir et de succès.

*** $*$

Nous conclurons en disant que la contradiction apparaît dans Topaze comme une idée constante qui prend sa source au niveau du titre et se répand dans chacun des langages paraverbaux employés dans la pièce dramatique, c'est-àdire: décors, costumes, accessoires, musique, bruitages et gestes. Deux contrastes physiques nous sont montrés, la belle et la bête, deux mondes opposés celui de l'enseignement et celui des affaires municipales, deux moments chronologiques, le passé et le présent, deux visions contradictoires de l'univers: celle de la fantaisie et celle de la cruelle réalité. 
On nous présente également deux espaces référentiels édifiés sur les plus belles contradictions: celui de l'enseignement, où Pagnol se meut à la perfection car par tradition familiale il lui appartient, où sont cultivés le savoir, la science, l'honnêteté, les sentiments et les valeurs humaines, mais qui est voué à l'échec, et celui des affaires où fleurissent l'égoïsme, le manque de conscience, la passion de l'argent, et qui triomphe.

Quant aux femmes, Suzy et Ernestine, elles se distinguent toutes deux par leur manque de moralité et leur excès d'intérêt personnel. Elles sont capables de se vendre au meilleur posteur ou d'utiliser les plus ignobles astuces afin de mener à bon terme leur ruée vers l'or. Si nous ajoutons à ces deux femmes vénales la toute puissante baronne, despote et insolente, nous pouvons conclure que Pagnol a mis dans Topaze une très légère couche de misogynie.

S'il est vrai que Topaze est une pièce comique et que plusieurs scènes provoquent le rire le plus fin, nous pouvons assurer aussi que derrière certains éclats se cache une philosophie extrêmement ironique. Le comique et l'ironie vont de pair dans l'oeuvre de Pagnol, elles sont complémentaires l'une de l'autre au point qu'il joue avec ces deux armes d'une façon si magistrale qu'il touche la sensibilité du lecteur, provoquant en lui la peine et la commisération de même que le sourire et le rire.

Même si tous les personnages font usage des mêmes procédés de communication, l'on se rend compte que celle-ci s'établit sur des fréquences différentes. C'est pourquoi le message a du mal à passer et que seul le froid langage des chiffres est compris de tout le monde.

En réalité, le message que veut transmettre Marcel Pagnol, en utilisant un personnage qui devient un type littéraire dès la publication du livre, c'est que les portes du succès et de la gloire sont ouvertes à toutes ces prétendues victimes qui, à un moment donne, savent faire usage de cette force sûre qu'est l'intelligence. 